\title{
Tecnologias não invasivas de cuidado ao parto normal: Percepção de puérperas
}

\author{
Non-invasive technologies of care in normal birth: Perception of puerperals \\ Tecnologías de cuidado no invasivas en parto normal: Percepción de puerperales
}

Recebido: 19/06/2021 | Revisado: 25/06/2021 | Aceito: 28/06/2021 | Publicado: 13/07/2021

\author{
Amanda Solar Martins dos Reis \\ ORCID: https://orcid.org/0000-0002-9170-6812 \\ Centro Universitário de Volta Redonda, Brasil \\ E-mail: amandasolar@uol.com.br \\ Mariana Calzavara Rodrigues \\ ORCID: https://orcid.org/0000-0001-6024-8890 \\ Centro Universitário de Volta Redonda, Brasil \\ E-mail: marianacalzavararodrigues@gmail.com \\ Mariana Vieira da Conceição \\ ORCID: https://orcid.org/0000-0002-0933-3819 \\ Centro Universitário de Volta Redonda, Brasil \\ E-mail: mariana_vieirasouto@ hotmail.com \\ Odete Alves Palmeira \\ ORCID: https://orcid.org/0000-0003-1487-1096 \\ Centro Universitário de Volta Redonda, Brasil \\ E-mail: odetepalmeira@hotmail.com \\ Rosane Belo Carvalho de Castro \\ ORCID: https://orcid.org/0000-0001-6744-7358 \\ Centro Universitário de Volta Redonda, Brasil \\ E-mail: rosane_b_c_c@hotmail.com \\ Renata Martins da Silva Pereira \\ ORCID: https://orcid.org/0000-0001-7642-6030 \\ Centro Universitário de Volta Redonda, Brasil \\ Universidade do Estado do Rio de Janeiro, Brasil \\ E-mail: renataenfprofessora@gmail.com
}

\begin{abstract}
Resumo
Esta pesquisa teve como objetivos descrever o uso de tecnologias não invasivas de cuidados utilizadas pela equipe de saúde no parto normal e identificar os benefícios referidos pelas puérperas após a utilização de tais tecnologias. Estudo descritivo, de abordagem quantitativa e desenvolvido através de pesquisa de campo. A coleta de dados foi realizada entre os meses de agosto e setembro de 2020, por meio de formulário do Microsoft forms, distribuídos às possíveis participantes pelas redes sociais. Participaram do estudo 213 puérperas maiores de 18 anos e com até um ano pós-parto. Os resultados apontam que: $54 \%$ das participantes tinham curso superior, $58 \%$ tiveram parto na rede pública, $71 \%$ tiveram acesso ao banho morno durante o trabalho de parto, $60 \%$ puderam escolher a posição durante o parto e 50\% utilizaram a bola suíça no pré-parto. Conclui-se que foram utilizadas tecnologias não invasivas de cuidado ao parto nas experiências das participantes e que seu uso ajudou no controle da dor durante este período. Ainda é necessário ampliar o uso destas tecnologias para o benefício das parturientes e para melhorar a qualidade e humanização do parto normal.
\end{abstract}

Palavras-chave: Tecnologia; Parto normal; Período pós-parto; Parto humanizado.

\begin{abstract}
This research aimed to describe the use of non-invasive care technologies used by the health team in vaginal birth and to identify the benefits reported by postpartum women after using such technologies. Descriptive study, with a quantitative approach and developed through field research. Data collection was carried out between the months of August and September 2020, using a Microsoft forms form, distributed to possible participants through social networks. A total of 213 mothers over 18 years of age and up to one year postpartum participated in the study. The results show that: $54 \%$ of the participants had a university degree, $58 \%$ gave birth in the public network, $71 \%$ had access to a warm bath during labor, $60 \%$ were able to choose the position during childbirth and $50 \%$ used the Swiss ball in the pre-partum. It is concluded that non-invasive birth care technologies were used in the participants' experiences and that their use helped to control pain during this period. It is still necessary to expand the use of these technologies for the benefit of parturients and to improve the quality and humanization of normal birth.
\end{abstract}

Keywords: Technology; Normal birth; Postpartum period; Humanized birth. 


\begin{abstract}
Resumen
Esta investigación tuvo como objetivo describir el uso de tecnologías de atención no invasivas utilizadas por el equipo de salud en el parto vaginal e identificar los beneficios reportados por las mujeres en el posparto después de utilizar dichas tecnologías. Estudio descriptivo, con enfoque cuantitativo y desarrollado a través de investigación de campo. La recogida de datos se realizó entre los meses de agosto y septiembre de 2020, mediante un formulario de Microsoft, distribuido a los posibles participantes a través de las redes sociales. En el estudio participaron un total de 213 madres mayores de 18 años y hasta un año después del parto. Los resultados muestran que: 54\% de las participantes tenía un título universitario, $58 \%$ dio a luz en la red pública, $71 \%$ tuvo acceso a un baño caliente durante el parto, $60 \%$ pudo elegir la posición durante el parto y 50\% utilizó el Balón suizo en el período preparto. Se concluye que en las experiencias de las participantes se utilizaron tecnologías no invasivas para el cuidado del parto y que su uso ayudó a controlar el dolor durante este período. Todavía es necesario ampliar el uso de estas tecnologías en beneficio de las parturientas y mejorar la calidad y humanización del parto normal.
\end{abstract}

Palabras clave: Tecnología; Nacimiento normal; Puerperio; Nacimiento humanizado.

\title{
1. Introdução
}

O nascimento é historicamente um evento natural e pode marcar a vida da parturiente de diversas formas, a depender do modo como for conduzido o trabalho de parto e parto. O momento do parto fica eternizado na memória por ser uma das maiores transformações físicas e psíquico-emocionais vivenciadas pela mulher. Sendo assim, é de extrema responsabilidade dos profissionais de saúde envolvidos na sua assistência respeitar a mulher e suas escolhas e garantir a ela, o acesso aos benefícios dos avanços científicos de qualidade, porém sem deixar de estimular sobretudo a autonomia feminina (Brasil, 2017).

Este estudo trata da percepção de puérperas sobre o uso de tecnologias não invasivas de cuidado utilizadas pela equipe de saúde em seu parto normal.

As tecnologias não invasivas de cuidado (TNIC) de enfermagem obstétrica foram definidas como o conjunto de técnicas, procedimentos e conhecimentos utilizados pelo enfermeiro durante sua relação de cuidado profissional, que, por sua concepção ecológica, compreende o parto como um processo fisiológico, respeitando sua natureza e a integridade corporal e psíquica das mulheres. Com a finalidade de deixar a mulher livre para ser protagonista de seu momento de parto, e auxiliar somente naquilo que for demandado pela mulher e sobre sua escolha (Nascimento, et al, 2010).

Antigamente, o parto ocorria no ambiente domiciliar, assistido por parteiras, familiares e curandeiras, mas devido à intercorrências médicas, o parto transferiu-se do domicílio para o ambiente hospitalar, deixando de ser um evento íntimo, privado e feminino, convertendo-se para um fenômeno intervencionista e público, excluindo a mulher do protagonismo do parto, em uma busca desmensurada de medicalização, visando a segurança da mulher e ao recém-nascido no momento do trabalho de parto. Segundo a Organização Mundial da Saúde, o aumento de medicalização no trabalho de parto e parto, afeta de forma negativa a experiência da mulher, porque enfraquece a sua capacidade para dar à luz e sua autonomia no momento do nascimento de seus filhos (OMS, 2018; Brasil, 2001).

Se por um lado, o avanço da obstetrícia contribuiu com a melhoria dos indicadores de morbidade e mortalidade materna e perinatais, por outro permitiu a concretização de um modelo que considera a gravidez, o parto e o nascimento como doenças e não como expressões de saúde, expondo as mulheres e recém-nascidos a altas taxas de intervenções, que deveriam ser utilizadas de forma parcimoniosa e apenas em situações de necessidade, e não como rotineiras (Brasil, 2017).

Diante disso, a Organização Mundial da Saúde (OMS) publicou em fevereiro de 2018, novas diretrizes acerca dos padrões de atendimento obstétrico afim de reduzir intervenções médicas desnecessárias, com a recomendação de não interferir no trabalho de parto, para que este seja acelerado, a menos que haja uma complicação evidente. A nova diretriz, incluiu 56 recomendações com baseamento científico acerca de quais cuidados devem ser administrados durante o trabalho de parto e parto, à mulher e ao bebê. Baseadas nos direitos humanos, enfatizou-se a importância de se ter uma experiência positiva de parto. As recomendações incluem, ser acompanhada por alguém de escolha da mulher, comunicação efetiva, garantir privacidade e integridade, técnicas de relaxamento, respiração, música, massagens entre outras (Who, 2018). 
Embora o trabalho de parto seja fisiológico, este é caracterizado por alterações mecânicas e hormonais que conduzem à dilatação do colo uterino, a passagem do feto pelo canal vaginal e sua expulsão para o exterior causando dor, ou algum grau de desconforto, dependendo de cada mulher a ser assistida (Brasil, 2011).

A dor que se origina do trabalho de parto, pode ser interpretada de diferentes formas, porque decorre de influências psíquicas, medo, ansiedade, experiências, culturas e constituição genética. Reconhece-se que a dor no trabalho de parto deve ser aliviada, visto que pode acometer prejuízos para a parturiente e para o feto. A equipe de saúde, bem como o enfermeiro obstetra tem a responsabilidade de oferecer uma assistência adequada, utilizando de métodos farmacológicos e não farmacológicos, para que a mulher consiga suportar a dor e tais desconfortos que são produzidos na parturição. A Teoria Interpessoal de Peplau, aponta que a enfermagem consiste em compreender o comportamento dos seres humanos, para que o profissional e o paciente, se ajudem, a identificar as dificuldades percebidas, e a aplicar os princípios de relação interpessoal aos problemas identificados, em todos os níveis de experiência (Franzoi, et al, 2016).

Os cuidados não farmacológicos são instituídos pelo Ministério da Saúde, propostos por meio do processo de humanização. Estes cuidados são opções beneficentes para alívio dor da parturiente durante o trabalho de parto, introduzidos de forma a substituir técnicas invasivas, analgésicas e anestésicas (Hanum, Mattos, Matão \& Martins, 2017).

Os métodos não farmacológicos, segundo à diretrizes nacionais de assistência ao parto normal, incluem oferecer imersão em água, apoiar técnicas de massagens e relaxamento, acupuntura podendo ser oferecida somente se houver profissional habilitada para tal, músicas de escolha da parturiente, aromaterapia, deambulação. Informações existentes nas literaturas, também reconhecem a bola suíça, cavalinho e quatro apoios como técnicas não farmacológicas para alívio da dor (Brasil, 2017).

Embora as tecnologias não invasivas de cuidado na assistência ao parto, sejam recomendadas, o seu uso ainda não é rotina, prevalecendo as intervenções desnecessárias. Com base nisso, as tecnologias não-invasivas de cuidado de enfermagem obstétrica (TNICEO), técnicas desenvolvidas pelas enfermeiras obstétricas, tem o objetivo de oferecer um cuidado à mulher onde ela possa ser a protagonista do seu próprio parto, desmedicalizar e humanizar no processo de parto e pós-parto (Vargens, et al, 2017).

Diante desta perspectiva de discutir o uso das TNIC, foi levantada a seguinte questão: Como se deu a utilização de tecnologias não invasivas de cuidados ao parto normal durante o trabalho de parto, na perspectiva de puérperas, e quais benefícios citados pelas mesmas frente ao uso destas tecnologias?

Diante do exposto, este estudo teve como objetivo descrever o uso de tecnologias não invasivas de cuidados utilizadas pela equipe de saúde no parto normal e identificar os benefícios referidos pelas puérperas após a utilização de tais tecnologias.

\section{Metodologia}

Foi realizada um estudo transversal, de cunho descritivo, com ênfase na utilização e utilidade das informações em saúde, na qual se contextualizaram as opiniões de puérperas do estado do Rio de Janeiro.

Os sujeitos do estudo foram puérperas maiores de 18 anos e que aceitaram os termos da pesquisa. Os critérios de inclusão foram ter tido um parto normal até um ano atrás e residir no estado no Rio de Janeiro.

Optou-se por utilizar ambientes virtuais para a realização do estudo, visto que na área de saúde representa uma possibilidade econômica, com maior velocidade de informação e produção científica, capaz de ultrapassar barreiras como idiomas, viabilizando a realização de estudos comparativos multicêntricos internacionais. Além disso, as pesquisas pela Internet proporcionam maior praticidade e comodidade às participantes do estudo, podendo resultar na melhora do número de respostas obtidas (Faleiros, et al, 2016).

As puérperas foram abordadas por meio da Internet, e convidadas a participar do estudo, após conhecerem os 
objetivos do mesmo. O instrumento para a coleta de dados, foi um formulário eletrônico on-line viabilizado por meio do aplicativo Microsoft forms ${ }^{\circledR}$, por meio do qual se viabilizou um formulário individual composto de perguntas relacionadas ao objetivo da pesquisa, de fácil e rápido acesso às participantes. Para a divulgação do instrumento, foram postados links do questionário em diversos ambientes virtuais como Facebook ${ }^{\circledR}$ e Instagram $^{\circledR}$, principalmente em grupos relacionados ao parto e direcionados a mães e recém-nascidos.

Os formulários foram disparados e acompanhados diariamente, por um período de 45 dias. Findo este prazo, contabilizou-se 283 participantes do estudo.

As perguntas abordaram o perfil das mulheres (idade, escolaridade, idade gestacional no momento do parto), se houve orientação do profissional de saúde sobre o parto durante seu pré-natal e se foram utilizadas as seguintes TNIC, em seu trabalho de parto: banho morno, escolha de posição durante trabalho de parto e parto, exercícios com a bola suíça, massagens, musicoterapia, aromaterapia, e outros que as mesmas quisessem citar.

A análise de dados foi feita por estatística descritiva, tendo os resultados apresentados em números absolutos e seus percentuais.

As participantes tiveram acesso ao Termo de Consentimento Livre e Esclarecido, e conheceram os objetivos da pesquisa e o caráter voluntário de sua participação, e assim deram seu consentimento no formulário. Atendendo à Resolução CNS/MS n 466, de 12 de dezembro de 2012 que trata de pesquisas com seres humanos, o estudo foi encaminhado ao Comitê de Ética em Pesquisa do Centro Universitário de Volta Redonda - UniFOA e foi aprovado sob número 4.078.043.

\section{Resultados e Discussão}

Retornaram da pesquisa 283 formulários preenchidos, sendo que após a primeira análise foram excluídos 70 participantes, por terem tido parto cesáreo, não dado seu consentimento para a pesquisa no formulário, ou ainda por ter deixado de responder perguntas sobre dados sociodemográficos, perfazendo ao final um total de 213 formulários com respostas válidas.

A idade das mulheres, variou entre 18 e 40 anos, sendo a prevalência de mulheres de 24 anos, quanto ao nível de escolaridade 8 (4\%) mulheres tinham o ensino fundamental, 89 (42\%) ensino médio e 116 (54\%) ensino superior. Quanto ao perfil clínico-obstétrico 31 (14\%) das mulheres responderam ter tido um parto de risco, e 194 (86\%) tiveram um parto de risco habitual.

Quanto ao local do parto, 123 (58\%) foram realizados em instituições de saúde públicas e 88 (42\%) em instituições privadas.

Quando perguntadas, se haviam recebido orientações sobre o momento do parto durante o pré-natal, 144 (68\%) das mulheres responderam de forma afirmativa, e 69 (32\%) das mulheres não tiveram as orientações sobre o momento do parto nas consultas de pré-natal.

Em contrapartida, se comparado à pesquisa de Almeida, Acosta e Pinhal (2015), pode-se perceber que houve um predomínio de 104 mulheres $(79,4 \%)$ de mulheres que declararam não ter recebido orientações sobre as metodologias não farmacológicas de alívio ao parto normal durante o pré-natal realizado em unidades básicas de saúde. As orientações sobre o parto e nascimento devem fazer parte das consultas pré-natais para auxiliar as futuras parturientes a tomarem decisões sobre a via de parto e propor um plano de parto adequado à sua realidade (Brasil, 2017).

Em relação aos recursos que as participantes tiveram a oportunidade de experimentar/utilizar no momento do trabalho de parto, $152(71 \%)$ das mulheres tiveram acesso ao banho morno. Esta prática também foi o método mais utilizado pelas mulheres no momento do trabalho de parto em outro estudo analisado (Dias, et al, 2018). Também na pesquisa de Almeida, Acosta e Pinhal (2015), o banho de chuveiro foi relatado como o método não farmacológico para alívio da dor do parto mais frequente entre as participantes, aparecendo em (53\%) dos relatos das puérperas, sendo o preferido e citado pelas mesmas 
como resolutivo.

Cabe destacar que na realidade brasileira, vivenciada por parturientes atendidas no Sistema Único de Saúde, torna-se comum a adequação de espaços para alocação de parturientes em trabalho de parto e nem sempre existe a estrutura e espaço físico para utilização de TNIC pela equipe de saúde. O banheiro com chuveiro de água aquecida ainda permanece como uma recorrência nas maternidades, e pode ser usado como estímulo para movimentação e minimização da dor no trabalho de parto.

No banho de aspersão/imersão, a água quente estimula a chegada da mensagem de forma mais rápido ao cérebro, do que o estímulo da dor, bloqueando a sensação dolorosa de forma efetiva. O calor também promove o aumento da circulação sanguínea e inibe os agentes estressores, motivados pela contração durante o trabalho de parto, devido ao contato com o tecido, é capaz de uma certa melhoria no metabolismo e da elasticidade, reduzindo o prelúdio da dor (Araújo, et al, 2018).

Quanto ao uso da técnica da bola suíça, 108 (50\%) mulheres tiveram acesso a este recurso. A bola suíça, também conhecida como bola do nascimento, promove o exercício da região pélvica, além de ser um método com baixo custo financeiro. Os exercícios com a bola durante a gravidez e o trabalho de parto, têm benefícios como o relaxamento e alongamento, correção da postura e o fortalecimento da musculatura (Araújo, et al, 2018; Barbieri, et al, 2013).

Embora algumas parturientes tenham medo e insegurança de utilizar a bola suíça, esta pode trazer relaxamento e bemestar com exercícios bem conduzidos e de fácil aplicação. É preciso que a equipe de Enfermagem esteja ao lado da mulher e dê apoio e segurança para utilização da bola com sucesso. Segundo a teoria de enfermagem de Peplau, que é centrada nas relações interpessoais, cabe ao enfermeiro ajudar o paciente a diminuir a ansiedade e convertê-las em uma ação construtiva no processo terapêutico, que auxilia no desenvolvimento pessoal tanto do profissional, quanto do paciente (Franzoi, et al, 2016).

Nas maternidades embora existam bolas suíças disponíveis, na prática ainda observa-se que as mesmas ficam expostas no pré-parto, porém sem uso rotineiro no manejo do trabalho de parto vaginal. É necessário que todos os membros da equipe de saúde envolvidos no cuidado às parturientes conheçam as indicações e benefícios dos exercícios durante os períodos clínicos do parto, para assim otimizá-los em sua assistência prestada às parturientes.

Com relação à liberdade de escolha da posição durante a dor, $130(60 \%)$ mulheres tiveram a oportunidade de optar pela posição de sua preferência. O agachamento no momento do trabalho de parto, foi utilizado por 101 (47\%) das participantes da pesquisa. Estes dados demonstram uma evolução no processo de assistir ao trabalho de parto no SUS e na rede privada. Pois atende às recomendações das Diretrizes Nacionais de Assistência ao Parto Normal, que preconizam a humanização da assistência obstétrica e a valorização da fala e dos desejos da parturiente no momento da parturição (Brasil, 2017).

Entretanto uma boa parte delas não teve seu direito de escolha preservado em relação a encontrar uma melhor posição e permanecer nesta para alívio da dor e autocontrole durante o trabalho de parto. Essa realidade ainda é vivenciada por muitas parturientes, que não encontram, na equipe que as atende durante o parto, apoio e alusão às práticas baseadas em evidências no momento de nascimento de seus filhos.

Na pesquisa de Ritter, Gonçalves \& Gouveia (2020), constatou-se que a prática de mudança de posição, foi relacionada com a melhor evolução do trabalho de parto e ao bem-estar materno e fetal, diminuição da sensação dolorosa e aumento da satisfação materna, principalmente quando praticadas as posições verticais e lateralizadas.

Quanto à prática de massagens durante o trabalho de parto, 103 (48\%) das mulheres tiveram acesso à esse recurso, que é um dos métodos não farmacológicos mais eficazes para redução da dor durante o trabalho de parto, pois tem como benefícios a promoção do relaxamento, o aumento do fluxo sanguíneo e consequentemente a oxigenação dos tecidos e reduz o estresse emocional, logo, todos esses benefícios se tornam fundamentais, para aliviar a dor que esta parturiente sente durante o trabalho de parto (Araújo, et al, 2018; Barbieri, et al, 2013).

Quanto à musicoterapia, 50 (23\%) mulheres puderam utilizar a música como recurso de alívio da dor. Essa tecnologia 
não invasiva, durante o trabalho de parto, é capaz de diminuir a ansiedade, aflição, estresse, medo e pânico da mulher e tem o intuído de promover o alívio da dor (Araújo, et al, 2018).

A equipe de saúde e de enfermagem deve proporcionar um ambiente favorável à boas experiências no momento do parto, e a música pode ser uma aliada para ajudar na concentração e proporcionar tranquilidade durante as contrações e no período expulsivo. Quando o cérebro da parturiente reconhece a música tocada, é possível notar que outras áreas do cérebro também são acionadas, bem como as partes responsáveis pelos movimentos, memória, atenção e emoção, quebrando assim, a tríade medo-tensão-dor (Aguiar, et al, 2019; Hanum, et al, 2017).

Com relação à aromaterapia, 38 (17\%) mulheres puderam fazer o uso desse método, que utiliza as plantas através de óleos essenciais, como por exemplo: lavanda, para ajudar na evolução do trabalho de parto e parto e no alívio da dor. Esse método pode ser utilizado por meio absorção na inalação ou no uso tópico na pele (Lehugeur, Strapasson \& Fronza, 2017).

O cavalinho foi citado por $26(12 \%)$ das mulheres como TNIC no parto e coadjuvante no processo de auxílio ao desenvolvimento do parto normal. Este método consiste em um equipamento semelhante a uma cadeira com assento invertido, onde a gestante apoia os braços e o tórax para frente, aliviando as costas. A gestante pode optar por ficar nessa posição para receber massagem na região lombar, durante as contrações, que tem o objetivo de relaxar a parturiente, e aliviar a dor (Araújo, et al, 2018).

Além dessas tecnologias não invasivas, 24 (11\%) mulheres usaram a cromoterapia no trabalho de parto, e 33 (15\%) mulheres não tiveram oportunidade de experimentar nenhum desses cuidados. O que reflete um número significativo de parturientes que ainda experimentaram uma condução do parto normal sem auxílio de TNIC, refletindo assim em sua experiência de parto de forma menos positiva.

Quando perguntado quais foram os efeitos desses cuidados durante o trabalho de parto em relação a dor, 147 (69\%) mulheres disseram que o uso de tais TNIC trouxe alívio da dor e bem-estar. Sendo assim, compreende-se que, para cada mulher em trabalho de parto, o padrão de dor sentida e referida deve ser avaliado de acordo com a necessidade de cada momento. Fisiologicamente, as contrações uterinas tendem a causar sensações dolorosas, principalmente durante o segundo e o terceiro período clínico do parto, sendo assim torna-se relevante oferecer TNIC às parturientes para auxiliar no controle da dor. É neste momento, que a assistência humanizada pode auxiliar, pois o apoio, as práticas de conforto e técnicas não farmacológicas de alívio da dor proporcionam à parturiente momentos de relaxamento e, com isso, há uma diminuição das sensações dolorosas entre um período e outro de contração (Sheen \& Slade, 2018; Hanum, et al, 2017).

Em estudo realizado com puérperas no sul do Brasil foi destacada a relevância da atuação dos profissionais como educadores em saúde, a fim de promoverem o conhecimento acerca das TNIC no parto normal, para empoderar as mulheres e, com isso permitir que decidam os caminhos relativos ao seu trabalho de parto de forma consciente sendo as protagonistas desse momento do seu viver (Santana, et al, 2020).

Um pequeno número, ou seja, 11 (5\%) não experimentaram benefícios com o uso dos métodos. De acordo com Gallo, et al (2011) podem existir várias razões para este acontecimento, visto que existem fatores que influenciam no momento como: os sentimentos e emoç̃es, a preparação para o parto, idade da paciente e até o tamanho do feto podem interferir no aceite e experimentação das TNIC durante seu processo de parturição.

Em contrapartida, outras $22(10 \%)$ mulheres alegaram não ter sentido nenhuma diferença com a utilização das TNIC durante o trabalho de parto e outras 33 (16\%) mulheres não tiveram ao menos a oportunidade de experimentar estes cuidados.

Desta forma o processo de parto deve ser tema de orientações e programas de educação em saúde para gestantes e parturientes, para facilitar a disseminação de informações e desmistificação do uso de TNIC, tanto pelas parturientes como pelos profissionais de saúde que as atendem. 


\section{Conclusão}

A utilização das tecnologias não invasivas de cuidado no parto normal são uma opção de cuidado de enfermagem e da equipe multiprofissional para assegurar um parto mais seguro para a mãe e o bebê, além de propiciar, em níveis individuais, conforto e bem-estar para a mulher.

As TNIC auxiliam na tomada de consciência da parturiente sobre seu papel de protagonista no ato de parir e permitem o desenvolvimento da autonomia e do autocuidado durante o trabalho de parto.

A maioria das participantes teve acesso ao banho morno durante o trabalho de parto, sendo seguido das tecnologias de cuidado: escolha de posição durante a parturição, uso da bola suíça, massagens na região lombar, musicoterapia, aromaterapia, uso do cavalinho e cromaterapia.

Este estudo aponta que a maioria das participantes teve acesso a algum tipo de TNIC, e relata como benefício do uso das tecnologias o alívio da dor no trabalho de parto. Um pequeno, porém relevante, número de mulheres não pode experimentar os benefícios das TNIC. Ainda, observou-se um pequeno número de mulheres, que não tiveram acesso a informações essenciais sobre o momento do trabalho de parto, sobre como este acontece e sobre os cuidados a que tem direito e estão disponíveis, podendo interferir diretamente na experiência do parto para a mulher.

Faz-se necessária a disseminação de informações sobre as TNIC entre as gestantes e parturientes, e a conscientização dos profissionais de saúde a respeito do diferencial que a utilização das TNIC podem trazer para a mulher durante o nascimento de seus filhos. Sendo assim, deve-se incentivar a prática e o desenvolvimento de novos estudos sobre tais tecnologias e encorajar suas aplicações em campo.

\section{Referências}

Aguiar, Y. M. N. F., Silva, V. S., Dias, A. S. \& Alves, N. C. M. (2019). Prática integrativa e complementar, a utilização da musicoterapia no trabalho de parto: uma revisão integrativa de bibliografias. Revista Caribeña de Ciencias Sociales (octubre 2019). En línea: https://www.eumed.net/rev/caribe/2019/10/musicoterapia-trabalhoarto.html//hdl.handle.net/20.500.1

Almeida, J. M., Acosta, L. G. \& Pinhal, M. G. (2015). Conhecimento das puérperas com relação aos métodos não farmacológicos de alívio da dor no parto. Revista Mineira de Enfermagem. 19(3): 711-717.

Araújo, A. S. C., Correia, A. M., Rodrigues, D. P., Lima, L. M., Gonçalves, S. S. \& Viana, A. P. S. (2018) Métodos não farmacológicos no parto domiciliar. Rev Enferm UFPE. 12(4): 1091-1096.

Barbieri, M., Henrique, A. J., Chors, F. M., Maia, N. L. \& Gabrielloni, M. C (2013). Banho quente de aspersão, exercício perineais com bola suíca e dor no trabalho de parto. Acta Paulista de Enfermagem. 26(5):478-84.

Brasil. (2001). Parto, aborto e puerpério: assistência humanizada à mulher. Ministério da Saúde, Secretaria de Políticas de Saúde, Área Técnica da Mulher. Brasília: Ministério da Saúde.

Brasil. (2017). Diretrizes nacionais de assistência ao parto normal. Ministério da Saúde.

Dias, E. G., Ferreira, A. R. M., Martins, A. M. C., Mirlene, M. J. \& Janine S. A. (2018) Eficiência de métodos não farmacológicos para alívio da dor no trabalho de parto normal. Revista Enfermagem Foco. 9 (2): 35-39.

Faleiros, F., Käppler, C., Pontes, F. A. R., Silva, S. S. C., Goes, F. S. N. \& Cucik, C. D. (2016). Uso de questionário online e divulgação virtual como estratégia de coleta de dados em estudos científicos. Texto Contexto Enfermagem. 25(4):e3880014.

Franzoi, M., Lemos, K., Jesus, C., Pinho, D., Kamada, I., \& Reis, P. (2016). Teoria das relações interpessoais de Peplau: uma avaliação baseada nos critérios de Fawcett. Revista de Enfermagem UFPE on line, 10(4), 3653-3661. https://doi.org/10.5205/1981-8963-v10i4a11140p3653-3661-2016

Gallo, R. B. S., Santana, L. S., Marcolin, A. C., Ferreira, C. H. J., Duarte, G. \& Quintana, S. M. (2011) Recursos não farmacológicos no trabalho de parto: protocolo assistencial. Revista Femina. 39(1): 41-48.

Hanum, S. P., Mattos, D. V., Matão, M. E. L. \& Martins, C. A. (2017) Estratégias não farmacológicas para o alívio da dor no trabalho de parto: efetividade sob a ótica da parturiente. Rev enferm UFPE.

Lehugeur, D., Strapasson, M. R. \& Fronza, E. (2017). Manejo não farmacológico de alívio da dor em partos assistidos por enfermeira obstétrica. Revista de Enfermagem UFPE Online. 11(12):4929-37.

Nascimento, N. M., Progianti, J. M., Novoa, R. I., Oliveira, T. R. \& Vargens, O. M. C. (2010). Tecnologias não invasivas de cuidado no parto realizadas por enfermeiras: a percepção de mulheres. Escola Anna Nery. 14 (3):456-461. 
Research, Society and Development, v. 10, n. 8, e31610817371, 2021

(CC BY 4.0) | ISSN 2525-3409 | DOI: http://dx.doi.org/10.33448/rsd-v10i8.17371

Prata, J. A., Ares, L. P. M., Vargens, O. M. C., Reis, C. S. C., Pereira, A. L. F. \& Progianti, J. M. (2019). Tecnologias não invasivas de cuidado: contribuições das enfermeiras para a desmedicalização do cuidado na maternidade de alto risco. Escola Anna Nery.

Progianti, J. M. \& Vargens, O. M. C. (2004) As enfermeiras obstétricas frente ao uso de tecnologias não invasivas de cuidado como estratégias na desmedicalização do parto. Esc. Anna Nery. 8(2):194-197

Ribeiro, I., Pereira, P., Gomes, I., Morais, J., Gouveia, M., Nascimento, M., Neto, F., \& Sales, I. (2018). Tecnologias não invasivas de cuidado: percepção das puérperas. Revista de Enfermagem UFPE on line, 12(8), 2129-2136. https://doi.org/10.5205/1981-8963-v12i8a236584p2129-2136-2018

Ritter, S. K., Gonçalves, A. C. \& Gouveia, H. G. (2020). Práticas assistenciais em partos de risco habitual assistidos por enfermeiras obstétricas. Acta Paulista de Enfermagem. 33

Santana, C. de S., Oliveira, A. M. N., Medeiros, S. P., Cardoso, V. M., Silva, M. R. S., \& Cezar-Vaz, M. R. (2020). Expectativas e sentimentos das puérperas acerca do trabalho de parto e parto. Research, Society and Development, 9(9), e375997076

Sheen K., \& Slade P. (2018). What do women fear about childbirth? Clin Nurs.27(13-14),2523-35.

Vargens, O., Reis, C., Nogueira, M., Prata, J., Silva, C., \& Progianti, J. (2017). Tecnologias não-invasivas de cuidado de enfermagem obstétrica: repercussões sobre a vitalidade do recém-nascido [Non-invasive technologies in obstetric nursing care: effects on newborn vitality] [Tecnologías no invasivas de cuidado de enfermería obstétrica: repercusiones en la vitalidad del recién nacido]. Revista Enfermagem UERJ, 25, e21717. https://doi.org/10.12957/reuerj.2017.21717

Who. (2018) Recomendations intrapartum care for a postive. Organização Mundial da Saúde. 